\title{
Adapting Communities That Care in Urban Aboriginal Communities in British Columbia: An Interim Evaluation
}

\author{
Tammy Stubley, Indrani Margolin, Marcela Rojas \\ School of Social Work, University of Northern British Columbia, Prince George, Canada \\ Email: stubley@unbc.ca,mrgolin@unbc.ca, Marcela.Rojas@gov.bc.ca
}

Received January $11^{\text {th }}, 2013$; revised February $19^{\text {th }}$, 2013; accepted March $2^{\text {nd }}, 2013$

\begin{abstract}
Copyright (C) 2013 Tammy Stubley et al. This is an open access article distributed under the Creative Commons Attribution License, which permits unrestricted use, distribution, and reproduction in any medium, provided the original work is properly cited.
\end{abstract}

\begin{abstract}
A considerable amount of research has been conducted on Aboriginal mental health and health promotion. However, implementation and impacts of culturally relevant health promotion strategies have not been equally addressed. This article provides an interim evaluation of Connecting the Dots, an innovative project designed to support and promote the mental health of Aboriginal youth and families in urban areas in British Columbia. Connecting the Dots adapted the Communities that Care (CTC) model, a prevention planning program promoting positive youth development and reducing risk factors that predict youth's future involvement in problem behaviors. This article devotes specific attention to the necessitated adaptations of the CTC model to promote cultural relevancy in urban Aboriginal communities. Evaluation findings suggest that Aboriginal communities can successfully adopt mainstream evidence-based programming, provided that programs permit adaptations to meet the communities' needs. For urban Aboriginal communities, programs must be re-conceptualized so that the linear, western delivery model is transformed to a holistic and circular implementation approach congruent with Aboriginal worldviews. In the Connecting the Dots project, inclusion of traditional Aboriginal practices and key Aboriginal representatives were among the most well received model adaptations. Evaluation participants reported that the adaptations made to the CTC framework have been critical to sustainability.
\end{abstract}

Keywords: Aboriginal; Community; Health Promotion; Mental Health; Prevention; Urban; Youth

\section{Introduction}

Connecting the Dots (CTD) is an innovative project designed to support and promote the mental health of Aboriginal youth and families in urban areas through the mobilization of community resources and by tackling identified risk and protective factors. The project is federally funded by the Public Health Agency of Canada (PHAC) and is spearheaded at the provincial level by the Canadian Mental Health Association BC Division (CMHA BC) and the British Columbia Association of Aboriginal Friendship Centres (BCAAFC). It is locally implemented through Friendship Centres and CMHA branches in three British Columbia urban Aboriginal communities: Kelowna, Port Alberni, and Quesnel.

The foundational purpose of the CTD project is to adapt the Communities that Care model to an urban Aboriginal Canadian context, ultimately addressing the mental health of young urban Aboriginal families. Communities that Care (CTC) is a prevention planning program based on two approaches to prevention science: promoting positive youth development and reducing risk factors that predict youth's future involvement in problem behaviors (Flynn, 2008).

In 2010, the first phase of funding for the CTD project commenced in Kelowna, the first of three urban Aboriginal communities in British Columbia (BC) to pilot CTD. The second phase of CTD commenced following the approval of funding for an additional four years. Subsequently, in 2011, two addi- tional urban Aboriginal communities embraced CTD, implementing the project in Port Alberni and Quesnel.

This article devotes specific attention to the necessitated adaptations of the CTC model to promote relevancy in urban Aboriginal communities. The implementation of cultural practices is one of the driving forces of the CTC model. It engages community partners in a comprehensive manner to combat the societal concerns that are faced by several Aboriginal communities. The challenges and successes of the CTD adaptation of the CTC model will be explored. The CTD program holds great potential to impact societal concerns such as dysfunctional behaviors among children and youth. Ultimately, the adaptations of the CTC model encourage collaborative relations with the urban Aboriginal communities, which in turn will assist with unraveling the societal problems they face.

One of the remarkable features of the CTD project is the innovative nature of the partnership between CMHA BC and BCAAFC. Linking Aboriginal service providers with the mental health sector dismantles silos. Subscribing to a cooperative and collective professional practice that conceives new ways of knowing and has the benefit of value exchange is a foundational change. In other words, this partnership creates pathways across various sectors and among these organizations, including the affiliated Friendship Centres and CMHA BC branches.

At the provincial level the CMHA BC Division is the sponsoring association of the joint venture, but decision-making occurs in collaboration between the organizations. The BCAAFC, 
an Aboriginal umbrella organization, serves 25 Friendship Centres throughout the province of British Columbia (British Columbia Association of Aboriginal Friendship Centres, 2012). The BCAAFC possesses a wealth of knowledge and expertise working with urban Aboriginal people. The Friendship Centres provide a number of quality services to Aboriginal communities including services related to employment, substance use, family support, legal services, and cultural retention. Although each center is as unique as the community it serves, the communities are united by a common bond: to improve the quality of life for Canadian Aboriginal people and, most importantly, to protect and preserve Aboriginal culture for the benefit of all Canadians.

Similarly, the CMHA BC Division and member branches have extensive knowledge in promoting mental health. CMHA is a charitable association that promotes the mental health of all and supports the resiliency and recovery of people experiencing mental illness through public education, community-based research, and influence on public policy, and in addition provides direct services and support. CMHA BC Division has provided more than 55 years of service to British Columbians and is the umbrella organization for a network of 19 CMHA branches across British Columbia (Canadian Mental Health Association, 2012).

In sum, the CTD project offers an urban Aboriginal Canadian context adaptation of the prevention program planning model known as Communities that Care (CTC). The project reaps the expertise of two reputable organizations in developing visions, strategies, and concrete tools to improve the mental health of urban Aboriginal people-and BC is home to the secondlargest Aboriginal population in the country (BC Stats, 2006). The project builds on the mandates, core competencies and values of the two main partners, the Canadian Mental Health Association BC Division (CMHA BC) and the BC Association of Aboriginal Friendship Centres (BCAAFC).

\section{Communities That Care}

CTD is a multi-year, multi-site research and community development project utilizing the Communities that Care (CTC) model, which was developed by Drs. J. David Hawkins and Richard Catalano at the University of Washington in Seattle. CTC is a prevention planning program grounded in research from a variety of fields, including public health, psychology, education, sociology, social work, criminology, medicine, and organizational development. By extension, this model serves as a medium for improved interdisciplinary discourse. In other words, it is clear that the CTC model crosses several disciplines, which inevitably can foster communication for improved outcomes. CTC's trademark is the reinforcement of positive predictors of health that shield and prevent youth from misconduct, leading to the absence of negative outcomes such as violence, substance use, delinquency, teen pregnancy, high school dropout, and mental health issues (Flynn, 2008).

CTC is a linear process designed to assess risk and protective factors of mental health. Based on three decades of scientific research including longitudinal studies carried out in North America, Europe and New Zealand, CTC founders identified themes in the data collected that revealed risk and protective factors that reliably forecast youth's future involvement in problem behaviors (Flynn, 2008). The CTC model is a collaborative, community-driven process that consists of five phases: getting started; organizing, introducing, involving; developing a community profile; creating a community action plan; and implementing and evaluating the community action plan (Flynn, 2008). A number of methods are used to recruit community members, including information meetings, email, community forums, word of mouth, and snowball technique. Through a collaborative approach, CTC engages all areas of the community, extending from school representatives to law enforcement, to city and business leaders, to faith community members, to youth and health professionals, all of whom share a unity of purpose: promoting healthy outcomes for youth. The core value of CTC is that behaviors can be influenced by addressing certain factors which either increase the risk of youth becoming engaged in delinquency or guard against involvement (Hawkins, Catalano Jr., \& Miller, 1992).

Before turning to the CTD project let's first review the literature on integrated approaches to service delivery in mental health for Canadian Aboriginal populations to learn whether any common themes exist in the approaches and results of previous projects with Aboriginal communities.

\section{Literature Review}

A great deal of research is available on the topics of Aboriginal mental health and health promotion. However, the implementation and impact of culturally relevant health promotion strategies is not equally addressed. Studies of health promotion strategies such as the Communities that Care (CTC) model fail to provide tangible examples of culturally relevant adaptations. This paper seeks to explore these topics by examining available literature on Aboriginal mental health, health promotion, and CTC, while considering how these areas of interest have served to inform the CTC cultural adaptation model known as Connecting the Dots (CTD).

\section{Aboriginal Mental Health}

The concept of Aboriginal mental health has been discussed in scholarship on such topics as the basis for knowledge concerning Aboriginal mental health and multidisciplinary perspectives on Aboriginal mental health (Kirmayer \& Valaskakis, 2009). Kirmayer and Valaskakis (2009) compilation of multidisciplinary perspectives on Aboriginal mental health provides the reader with an opportunity to explore the various social, economic, and political issues affecting the health and wellbeing of Aboriginal people in Canada. McCormick's chapter, “Aboriginal Approaches to Counselling” (Kirmayer \& Valaskakis, 2009: pp. 337-354), depicts innovations in counseling approaches to working with Aboriginal populations. McCormick succinctly captures the essence of a well-informed and conscientious journey towards culturally competent practice. The chapter's content also serves to validate integrated approaches to promoting the health and well-being of Aboriginal clients. The author's assertion that additional research is needed to further establish the value and impact of both traditional and mainstream approaches serves as a challenge to the professional community to extend the body of knowledge in this area.

Other chapters explore factors influencing Aboriginal health and well-being, including Aboriginal worldviews, spirituality, tradition, and culture. The role of mainstream mental health methodologies and traditional healing practices in supporting the mental health of Aboriginal clients is also considered. The efficacy of amalgamating mainstream and traditional approaches 
is discussed, giving consideration to their applications within the context of multiple counseling scenarios, including sex abuse, career/vocational, suicide, and substance abuse (Kirmayer \& Valaskakis, 2009).

The histories, worldviews, and needs of Aboriginal populations have prompted acknowledgment in multiple fields of research and practice of the importance of developing and implementing services that reflect the uniqueness of Aboriginal culture. The development of Aboriginally relevant counseling methods is reflected in recent research efforts, such as Limb and Hodge's (2011) contemplation of the therapeutic implementation of spiritual ecograms, a unique assessment tool that incorporates the fundamental aspects of a genogram, with additional consideration given to past and present functioning, while considering the physical and spiritual aspects of the self.

Walker, Cromarty, Kelly, and St Pierre-Hansen (2009) offer a recent Canadian example of culturally competent service delivery in addressing the health needs of Aboriginal populations. Their introduction provides a helpful review of Aboriginal perspectives on well-being and healing, allowing the reader to better understand the difference between Aboriginal and mainstream perspectives. The comparative review provides particular insight into how integrated methods complement and strengthen the individual components while promoting improved client outcomes. The authors' discussion of existentialism and its homogenous roots in both mainstream and traditional ideologies concerning the search for meaning facilitates the consideration of not just the differences, but also the important similarities between the two belief systems.

\section{Health Promotion}

Various scholarly inquiries have been undertaken into the conceptualization and applicability of health promotion. These efforts have sought to discern the epistemological origins of health promotion strategies and their impact on communities and individuals. Several scholarly efforts have contributed to this body of knowledge (O’Neil, Pederson, Dupéré, \& Rootman, 2007; Rice, 2011).

The concept of health promotion is explored in Wendy Rice's Health promotion through an equity lens: Approaches, problems and solutions (2011). The author contends that global disparities in health equities have prompted a shift towards a more holistic, preventative approach to the delivery of health services. Additional assertions are made concerning traditional methods for addressing health-related concerns, which have been limited in their efficacy because of approaches that assume a disease management, reactive stance that fails to consider preventative measures for promoting health.

Yet another significant limitation in traditional methods is inequality in the delivery of health services, including in accessibility and quality of care for marginalized populations. Rice (2011) further asserts that traditional approaches are limited by their failure to adopt ecological methodologies. Ecological methods are described as processes that foster inclusive and holistic supports and interventions that consider the individual within the context of his or her local and global environments. Health promotion strategies are believed to support this ecological approach while seeking to effect change at various levels, including research, policy, and service delivery (Rice, 2011).

Rice reinforces the assertion that health promotion efforts support means of action that acknowledge the individual as a complex being who is not isolated from his or her environment. Further credence is given to a conceptualization of health promotion as a process empowering individuals to gain a better understanding of the factors that have a direct impact on their health and to better equip them to improve their state of well-being. Rice's analysis effectively conveys the value of health promotion strategies in supporting communities to take a localized stake in the improvement of structural supports and resources while promoting individual buy-in and accountability.

Health promotion's ecological approach was the focus of O'Neil et al., Health promotion in Canada: Critical perspectives (2007). The authors describe the ecological approach to health as a framework of action that considers the complexities of the systems that influence the overall health and well-being of individuals. These systems encompass micro, mezzo, and macro level transactions. Transactions may include environmental, community, and global factors. Furthermore, the ecological approach to health promotion is said to foster a social ecological stance on "the social, organizational, and cultural components of the environment” (O’Neil et al., 2007: p. 49).

The O'Neil et al. (2007) examination of the ecological approach to health promotion complements the existing body of knowledge on this topic and enhances understanding of health promotion's epistemological foundation. It is this foundation that gives further credibility to health promotion as a holistic approach to improving health and well-being. The inclusion of specific examples of the application of this approach would have served to strengthen the authors' stance. Furthermore, a comparative analysis of health promotion approaches and traditional methods would have supported understanding and appreciation of the merits of the model of choice.

\section{The Communities That Care Approach}

In his 2008 publication "Communities that Care: A comprehensive system for youth prevention and promotion, and Canadian applications to date," Robert J. Flynn explores the theoretical basis of Communities that Care (CTC) and its applications within the Canadian context. Flynn references the system's creators, Hawkins and Catalano Jr. (2002), and outlines their description of CTC as a functional approach that supports communities in strategizing the prevention of widespread acute behavioral trends among children and adolescents. The Social Development Model is identified as the theoretical foundation for CTC. Key health prevention principles, such as guided mobilization, multiple levels of intervention, and high fidelity, are a foundation for the CTC approach. Community readiness, training, and technical supports are described as key factors in determining the successful implementation of CTC.

Data are collected via a survey tool, designed especially for CTC to assist in gathering pertinent information from the selected population, typically comprised of children and youth actively enrolled in the public school system, and with a focus on risk and protective factors as determinants of acute behavioral trends among children and adolescents. The information collected is intended to inform and guide the community in selecting the most suitable evidence-based policies and programming. In addition, the CTC approach relies on a multiplephase strategy of five distinct stages: community readiness, community mobilization, data collection, findings review, and 
action plan implementation. Flynn (2008) further explains that a milestones and benchmark tool is utilized to determine the fidelity with which the CTC phases are implemented. Milestones are the community goals, while benchmarks are described as the actions the community assumes to meet these goals.

Flynn (2008) then describes the results of the CTC evaluation process. The reliability and validity of the youth survey tool are considered. The implementation of CTC in the State of Washington is reviewed, with emphasis given to the long-term impact on target communities. The data revealed improved inter-agency collaboration and coordination of services, enhanced community stakeholder involvement, improved access to educational opportunities, better adapted prevention strategies, and reduction in service duplication. The author gives additional consideration to CTC implementation outcomes in Pennsylvania, with stakeholder involvement, prevention knowledge, coalition function, community readiness, and barriers identified as key domains of measurability. Overall, the project has been effective throughout its target communities in reducing risk factors and acute behavioral trends among juvenile populations (Flynn, 2008).

Flynn's (2008) examination of CTC concludes with a review of approach implementations in Canada to date. Squamish, British Columbia, is identified as the first Canadian community to implement CTC. Described as a successful example of CTC implementation and sustainability, Squamish is regarded as a valuable source of information and support for other CTC target communities. Flynn goes on to describe his personal involvement in the implementation of CTC in three Ontario communities, including adapted variations of the approach. He concludes by giving consideration to the successes and shortcomings of CTC in the Canadian context.

The article provides a detailed review of the origins, the theoretical foundation, and impact of the CTC approach in the United States and Canada. Flynn (2008) provides the reader with the opportunity to learn about CTC and to consider the implications of implementing this approach at both the local and national levels. While the author's scope provides a comprehensive overview of CTC, little information is provided on the types of and reasoning behind the adaptations to the CTC approach in Canada. Flynn's title implies that CTC implementation within the Canadian context will be the focus of the review, but he provides little more than a quick overview of Canada's experience with the program. The intent of the present article is to contribute to the body of knowledge available on Canada's experience with the CTC model and to address the neglected issue of culturally relevant adaptation of CTC. In providing a more detailed analysis of a specific implementation of CTC in the Canadian context and in assessing the challenges and benefits of adapting CTC to Aboriginal approaches, this article furthers the development of models for health promotion in Aboriginal communities.

\section{Connecting the Dots: A CTC Adaptation for Urban Aboriginal Communities}

Health promotion initiatives support holistic and preventionfocused approaches to the delivery of health care. These holistic processes coincide with traditional Aboriginal approaches to health and healing. CTC is a public health strategy that embodies health promotion priorities such as community engagement and development. CTD is a recent CTC adaptation supporting a community development approach that promotes culturally relevant ways to engage the Aboriginal community in promoting the health and well-being of its members, while being cognizant of Aboriginal worldviews and priorities. In its integration of the holistic tenets of health promotion and traditional Aboriginal approaches to healing, CTD offers an exciting opportunity to develop culturally relevant health promotion strategies in Canada.

\section{The CTD Methodology}

CTC seeks to mobilize multiple social supports within the community to promote surroundings that foster positive mental health. The CTD project recruits and retains leadership by and for Aboriginal people. Aboriginal people are among the most studied populations in the world, and research with these groups has rarely been to their benefit (Smith, 1999). Moreover, historical research conducted on Indigenous peoples was based on factors that were not ethically sound, further perpetuating colonial power (Cochran et al., 2008). Therefore, it is crucial to adapt the CTC model, where necessitated, to cultivate cultural relevancy, practical success and, most importantly, Aboriginal community investment. In the absence of Aboriginal support and participation the program will not be effective, deflating its sustainability.

The teams endorsing the CTD project are cognizant of the impact of past research and they are hyper-vigilant to this sensitivity, ensuring a balance of culture, purpose, and ethics. CTD members recognize the value and importance of the project's aim, which drives the team to produce a promising foundation rooted in cultural awareness and safety-embracing the voice and expertise of the urban Aboriginal communities.

The CTD project applies a promising methodology informed by qualitative inquiry and guided by a Participatory Action Research (PAR) framework to mental health promotion focused on young Aboriginal families. CTD was designed to be highly collaborative, relevant, and empowering for all members involved. Involvement beyond a single community organization has the potential to impact those at greatest risk (Johnson, 1986). CTD clearly connects several organizations, which is extremely useful as it sheds insight on the problem of youth misconduct.

CTD is based on collaborative practice, relationship building, and information exchange achieved via community engagement, which led to community meetings to discuss barriers and partnerships, and to gain a better understanding of the community. The local project coordinators invested an enormous amount of energy in order to cultivate the relationships. Information exchange occurred with other CTC projects in British Columbia in order to facilitate best practice sharing and dialogue about how to overcome challenges. Because of bridging of wellknown community access points with key informants, services that were well known to others in the community have now become common knowledge to the key informants.

In the initial phases of CTC, one of the CTD teams advocated for an adaptation in the intervention training. Initially, an external, non-Aboriginal CTC trainer provided the training for the local community members. On closer inspection, it was agreed that it was essential for the training to be delivered from an Aboriginal perspective, by individuals with a lived experience and passion for Aboriginal ways of knowing. Subse- 
quently, the team contracted with Aboriginal CTC trainers from Oregon.

In addition to the hiring of Aboriginal trainers, one of the communities created an Elders' Guiding Circle to assist with the sharing of knowledge, traditions, and teachings. Prayer time at all project-related functions is another example of honoring Aboriginal traditions. Furthermore, the CTC survey questions were revised to make them more culturally applicable and relevant to the community. Conformity was reached in regards to developing cultural competency through formalized training and adopting project structural standards that reflect Aboriginal perspectives.

The CTD team developed "storytelling templates" to facilitate communication among key personnel and local partners while utilizing Indigenous methodologies. The written stories captured monthly through a storytelling template highlighted the project activities and tasks completed by the target community, and were reported on a quarterly basis. Some of the project activities were key leader training, recruitment of board members, workshops, building relationships with diverse community partners and, in addition, the building of relationships between mainstream mental health services and the urban Aboriginal community. The storytelling format was meant to reflect the integration of traditional Aboriginal perspectives and values with mainstream evaluation methodologies. The documents were reviewed and served to substantiate the adaptations of the model and triangulated information from interviews.

\section{Evaluation of CTD}

\section{Evaluation Process}

This paper explores the adaptations of a mainstream evidence-based model (CTC) implemented in three Aboriginal communities in British Columbia, focusing specifically on the findings from Phases I and II during the period 2009-2012. The evaluation process was guided by the project's research questions and objectives. The overall research question for this evaluation is:

- How effective is Communities that Care as a model to improve the mental health of urban Aboriginal youth in Canada?

The following has been identified as the secondary research question:

- What adaptations to the CTC model are required to improve its efficacy with urban Aboriginal communities in Canada?

\section{Data Collection Methods}

Evaluation focused on the analysis of information gathered through semi-structured interviews and storytelling templates. The process of arranging the CTD evaluation interviews and obtaining interviews with key participants relied on the efforts and expertise of local project coordinators. A purposeful sample was applied for the evaluation of the pilot studies. Participation in the interview evaluation of the CTC process was completely voluntary. Individual and dyadic interviews were conducted utilizing a semi-structured format. The semi-structured interview approach was adopted to allow for a rich dialogue, granting the interviewee the flexibility to explore their thoughts and feelings concerning the project. This technique allows for greater flexibility to make adjustments in order to accommodate the participant's needs and to support the disclosure of difficult and at times emotional information (Selltiz, Jahoda, Deutsch, \& Stuart, 1967). A semi-structured interview allows the interviewer and respondents to engage in a formal process that is guided by a set of questions to be addressed yet permits the inclusion of free-flow dialogue, allowing for a more thorough understanding of the subject matter (Cohen \& Crabtree, 2008). Interviews were guided by open-ended interview questions, which allowed the interviewers to maintain a balance between flexibility and control.

The provincial Project Liaisons, local Project Managers, Project Coordinators and Administrators, and Key Leader/ Community Board members were interviewed. All interviews were recorded and later transcribed. Face-to-face interviews were the preferred approach, although geographic and scheduling limitations required additional flexibility resulting in some of the interviews being conducted via teleconference. Interview participants were provided with an Information Letter and Consent Form. Twenty-five interviews were completed among all three pilot sites.

\section{Evaluation Methodology}

A Participatory Action Research (PAR) framework was employed to assess the work of the CTD project. PAR is a means of bridging the gap between researchers and stakeholders through collaborative endeavors that inform all aspects of the research process. PAR promotes a greater level of participation and influence by stakeholders, as compared to more traditional research processes (Turnbull, Friesen, \& Ramirez, 1998). This attractive feature resonated with the CTD team, as it is congruent with Aboriginal approaches. The CTD team collaborated closely with all participants involved during all phases of the evaluation process.

The evaluation process was also guided by exploratory and descriptive research approaches. The exploratory research approach is concerned with increasing knowledge about a relatively unknown topic (Singleton \& Straits, 2005). The descriptive research approach seeks to describe a phenomenon through a systemic and precise approach to data gathering (Singleton \& Straits, 2005). One of these tactics is the interview approach discussed earlier, which provided a better opportunity to consider the soundness of the information being gathered, by allowing the observer the opportunity to study the circumstances in which responses are provided. These qualities supported the decision to adopt semi-structured process interviews as the primary data-gathering tool.

A Thematic Content Analysis (TCA) approach will be adopted and applied in the examination of research findings. TCA is described as a "descriptive presentation of qualitative data” (Anderson, 2007: p. 1). TCA supports the depiction of themes, as identified within the research data. TCA is believed to provide an objective approach to data analysis (Anderson, 2007). Furthermore, thematic analysis offers an adaptable method for recognizing, studying, and exposing patterns contained in the data (Braun \& Clarke, 2006: p. 79).

\section{Findings}

Thematic analysis of the data collected from the semi-structured interviews and the storytelling templates yielded a wealth of insight concerning the project's efforts, successes, challenges, and overall progress. Interviews were conducted with the fol- 
lowing members: the Provincial Project Liaisons, Local Managers, Local Coordinators, administrative staff, and Community Board members. In addition, a document review of the quarterly storytelling templates was conducted. The themes will be structured in accordance with the overarching goals of the project:

- To initiate and sustain a long-term partnership between CMHA and Friendship Centres.

- To improve urban Aboriginal mental health.

- To adapt the Communities that Care model in an urban Aboriginal context.

After the data were analyzed, several themes emerged. The following section provides a summary of the following themes: Collaboration, Partnership, and Relationships; Cultural Awareness and Adaptations; Personal and Professional Lessons Learned; and Challenges, Barriers, and Time.

\section{Collaboration, Partnership, and Relationships}

The provincial partnership is described as progressing in mutually identified areas of interest, including collaborative practice, relationship building, and information exchange. In regards to successes, organizational information exchange has fostered improved common understanding, establishing the successful integration of traditional Aboriginal approaches and mainstream mental health principles. The CTD project has paved the way for CMHA BC and BCAAFC, as they were not formally affiliated in a working relationship of this nature. This type of success and growth creates a ripple effect, uniting many service providers and community members in an effort to support Aboriginal youth. As one Local Manager stated, "Connecting the Dots is exceptional in that it links the non-Aboriginal community and the urban Aboriginal population”. In describing the evolution of the partnership between the two provincial organizations, there was consensus among all of the participants that a key aspect of the process is that of "building relationships" and "building trust". The Local Managers for the project had an abundance of information to share on trust and partnership building. A Local Manager articulated:

I think what's unique is that it's forced two agencies that definitely had a relationship, but now it's forced us to work together. Each partnership like this helps strengthen the position of trust in terms of the people working at our agency. This project has expanded our organizational viewpoint, and you're right, it's the community viewpoint.

All of the respondents expressed satisfaction that the project is starting from a place where there are very limited services available for urban Aboriginal youth in terms of mental health. Acknowledging that this is a deficit within the communities promotes great discussion and the generation of ideas. A Provincial Project Liaison commented: "I really want this to be something that the community has ownership over. I want everyone to honor and respect everything that they come to the table with, and I feel like that's been very successful."

Jamieson et al. (2012) describe ten essential principles when conducting research with Indigenous communities, including creating an "open and transparent relationship with key community groups” (Jamieson et al., 2012: p. 16). This type of communication is the spirit of the Connecting the Dots project. Relationship building is an effective method for improving the community's capacity to understand Aboriginal mental health
(Jamieson et al., 2012).

One of the most meaningful contributions of this partnership is the bridging of well-known community access points with key informants. The CTD's partnership framework has "raised awareness" and has facilitated a rich discussion regarding mental health in the Aboriginal context. A Community Board Member reports that the partnership established has generated the conception of a "collective group energy," which fuels ideas and moves action forward. Bringing "people together with a clear purpose” was described as yet another example of successful collaboration. The project has successfully facilitated the process of people working and consulting together to support the youth in their community. Not surprisingly, the data reflect the project's focus, driven by such ingredients as collaboration, partnership, and relationship building in all three sites in British Columbia.

\section{Cultural and Awareness Adaptations}

The project adaptation successes were described by program participants as localized efforts to adapt the CTC model to the unique needs of each of the target communities, while adhering to the principal objectives of the project. Comments from the participants revealed that cultural awareness is fundamental in terms of the project's sustainability. This discovery has significant implications for the CTC model, ultimately making adaptations crucial to meet Aboriginal communities' needs. Jamieson et al. (2012) attest that "flexibility in study implementation while maintaining scientific rigour" (p. 17) may be necessary based on the needs of the community. Participants identified the adaptation of the model's training component from a "linear" form of delivery to a more "circular" approach as an example of a provincial level success. This distinction is important for several reasons: the inclusion of Aboriginal trainers strengthened the connection to the community, and the trainers highlighted the efficacy of the model as they shared personal successes about societal problems that were addressed in their own communities with the application of this model. As well, the curriculum was taught in a fashion that was complementary and congruent with the learning needs of the communities. One Local Coordinator reported:

Taking this model and applying it with a very distinct population and also what hasn't been done a whole lot particularly in Canada is looking at the mental health aspect of what can communities do in terms of promoting mental health... It is innovative. I definitely can see the connection with innovation strategy... It's a golden opportunity right now... CMHA [can] really deepen our understanding, our breadth of knowledge about this population of urban Aboriginal people who in many ways seem to be invisible...there's a lot of assumptions about Aboriginal people...

Project participants also celebrate the fact that all three sites hold a contract with two Aboriginal women from Oregon, one of them one of the original certified CTC trainers involved with the University of Washington in the first CTC research trials in the United States.

The process of promoting cultural competency has reportedly been enhanced through online access to educational opportunities. One of the Community Board members articulated: 
The project is described as "culturally driven". The project's approach to promoting cultural knowledge and skill is described as "very respectful to Aboriginal people". The development of cultural knowledge is reportedly supported by the project's networking opportunities. The project's positive, welcoming, and organized approach reportedly fosters a safe environment in which to explore culture.

In summary, the inclusion of traditional Aboriginal practices, such as the Elders' Guiding Circle and talking circles, and the revisions of the survey are described as some of the ways in which the project has been adapted in order to meet the needs of the Aboriginal communities. As the project progresses through the various phases of the CTC model it provides an opportunity to make additional adaptations that embrace the needs of the community while maintaining fidelity.

\section{Challenges, Barriers, and Time}

Evidence-based programming is a key aspect of the CTD model and one that is limited in capturing cultural relevancy. Ensuring "fidelity while implementing adaptations" is a primary challenge, according to one of the provincial Project Liaisons. The cultural component is fundamental to sustainability. Both Provincial Project Liaisons agreed that this is an important factor. For example, as one Provincial Project Liaison asked:

How do you maintain fidelity so that you can still have successful outcomes but also have a sustainable project that the community buys into in the long term so that once funding is over, the community is able to continue it.... So that's one of the major things that PHAC is looking at, so how do you navigate the implementation quality gap?

It should also be mentioned that during the initial phases of the project it was discovered that there was a lack of consistency in the level of knowledge and skill set concerning cultural competence, which later proved to be a significant challenge. Local Coordinators revealed an interesting finding, explaining that, "urban Aboriginal people have no problem accessing services". One Local Coordinator argued that people move from the reserve to the urban environment to improve their life situations. So, she continued,

The problem isn't for them accessing service but the people who are offering those services are providing challenges.... Because they're not aware of what the history is or why maybe a person can't provide a birth date or social insurance number or a fixed address.

The cultural competency component, one of the greatest contributions this project has made to the communities, in part grew out of this barrier. One of the lessons learned early on was the importance of focusing on cultural awareness and how it impacts the target population group. With this recognition the coordinators were driven to create cultural competence for other agencies to ensure their practices are culturally sensitive. This was not part of the Communities that Care framework, but it was beneficial to the project and the community to raise cultural awareness across agencies. Hence, many training events occurred with an overwhelming response from community partners. As a result of the feedback, it was clear that many participants left with a new level of cultural awareness. Chang- ing the training from the CTC model's "linear" form of delivery to a more "circular" approach was provided as an example of successful adaptation. The up side to this challenge is that front-line service providers are afforded the opportunity to look at their practices and reflect on how they can ensure urban Aboriginal clients receive their services and how the service providers can be of aid while simultaneously meeting funder requirements of demographics. It was clear in the data that the project coordinators are instrumental in opening up that dialogue and helping service providers become more proficient in cultural competency through ongoing dialogue, community engagement, and education opportunities.

Another hurdle the project must tackle is overcoming skepticism and mistrust derived from a history of programming aimed at effecting significant change in Aboriginal communities but often resulting in disappointing outcomes for the community. Additional challenges faced are issues of inclusion, tokenism, trust, and ambivalence on the part of the Aboriginal community. There is no denying that these issues were born of previous unethical research that was conducted among Aboriginal people. Aboriginal people have been submissive subjects rather than "participants", leading to "misinterpretations" as their voices were absent and an atmosphere was not created for possible modification of the data (Holmes, Stewart, Garrow, Anderson, \& Thorpe, 2002: p. 1268). Often conflict arises between traditional Aboriginal perspectives and mainstream ideologies, specifically concerning time sensitivity in lieu of mainstream time-lines and priorities. Participants identified workload issues, pressures, and scheduling restrictions as barriers to participating in the project.

\section{Conclusion}

According to the information collected throughout the evaluation process, the overall findings show that partnerships between the agencies and individual relationships are deepening and that this cohesion is resulting in creative and effective completion of the tasks and activities of the Communities that Care model. All participants expressed an enormous amount of appreciation, respect, and support for each role and member involved in the project. The project has brought together two provincial organizations and has the potential to bring organizations together on a national scale in the future. In Kelowna, Quesnel and Port Alberni, it has linked together the non-Aboriginal community and the urban Aboriginal population in an unprecedented way. The inclusion of traditional Aboriginal practices and key Aboriginal representatives were among the most well received model adaptations. Interview participants reported that the adaptations made to the CTC framework have been critical to sustainability. Current structural supports appear to be an essential aspect of the project's ongoing success.

Findings from the evaluation suggest that mainstream evidence-based programming can be successfully adopted by urban Aboriginal communities, with the strong caveat that programs must permit adaptations to meet the communities' needs. To execute an evidence-based program powered by a "one size fits all” approach is inadequate for urban Aboriginal communities. Essentially, such programs require re-conceptualization to transform a linear, western delivery model to a holistic and circular implementation approach, which is congruent with Aboriginal world views. Using a qualitative approach such as PAR provides a platform for work with the communities. It 
stimulates reflection and ongoing assessment of the successes and challenges, and it provides a venue to problem solve. Furthermore, the model presents as an effective medium for improved interdisciplinary discourse, while offering promising potential towards impacting societal concerns, such as dysfunctional behaviors among children and adolescents. In addition, by examining the adaptations in the context of the CTC model, PAR addresses whether the intended actions compromise fidelity. Overall, these findings support a strong foundation to maintain efficient continuance towards the completion of the current phase of the project. The Evaluators are confident that this project can succeed with ongoing consultation with Community that Care experts, coupled with strong knowledge and practices that support and honor Indigenous peoples.

\section{Acknowledgements}

First and foremost, we would like to honour and acknowledge all the First Nations territories upon which this project occurred. We are very thankful to the Canadian Mental Health Association (CMHA), BC Division and the BC Association of Aboriginal Centres for the implementation of this project through the local Friendship Centres and CMHA branches in three British Columbian urban Aboriginal Communities: Kelowna, Port Alberni and Quesnel. The authors wish to express their gratitude to the Provincial Project Liaisons, Trixie Ling and Fernando Polanco who offered support throughout the project. Special thanks to the Local Project Managers Trevor Barnes, Sandy Brunton, Bob Hargreaves, Cyndi Stevens, Shelagh Turner and Cam Martin for their guidance. Deepest gratitude is also due to the Local Project Coordinators Sheila Lewis, Amanda Swoboda, Lanny Kipling, Michelle March, Diandra Jurkic-Wells, and Shan Jacob Ross. Without their knowledge and commitment the project would have not been successful thus far. Finally, an honourable mention goes to the community participants known as the Key Leaders and the Community Board Members.

\section{REFERENCES}

BC Stats (2006). Aboriginal profiles of British Columbia: 2006. URL. http://bcstats.gov.bc.ca/StatisticsBySubject/AboriginalPeoples/Censu sProfiles/2006Census.aspx

Braun, V., \& Clarke, V. (2006). Using thematic analysis in psychology. Qualitative Research in Psychology, 3, 77-101. doi:10.1191/1478088706qp063oa

British Columbia Association of Aboriginal Friendship Centres (2012). About us. URL. http://www.bcaafc.com/initiatives/health

Canadian Mental Health Association (2012). How we can help: Connecting the Dots. URL.

http://www.cmha.bc.ca/how-we-can-help/aboriginal-families/connect ingthedots

Cochran, P. A. L., Marshall, C. A., Garcia-Downing, C., Kendall, E.,
Cook, D., McCubbin, L., \& Gover, R. M. S. (2008). Indigenous ways of knowing: Implications for participatory research and community. American Journal of Public Health, 98, 22-27. doi:10.2105/AJPH.2006.093641

Cohen, D., \& Crabtree, B. (2008). Semi-structured interviews. Robert Wood Johnson Foundation Qualitative Research Guidelines Project. URL.

http://www.sswm.info/sites/default/files/reference_attachments/COH EN\%202006\%20Semistructured\%20Interview.pdf

Flynn, R. J. (2008). Communities that care: A comprehensive system for youth prevention and promotion, and Canadian applications to date. URL.

http://www.socialsciences.uottawa.ca/ipc/pdf/8_IPCR2\%20-\%20Fly nn.pdf

Hawkins, J. D., \& Catalano Jr., R. F. (1992). Communities that care: Action for drug abuse prevention. San Francisco, CA: Jossey-Bass.

Hawkins, J. D., Catalano Jr., R. F., \& Miller, J. Y. (1992). Risk and protective factors for alcohol and other drug problems in adolescence and early adulthood: Implications for substance abuse prevention. Psychological Bulletin, 112, 64-105. doi:10.1037/0033-2909.112.1.64

Holmes, W. W., Stewart, P., Garrow, A., Anderson, I., \& Thorpe, L. (2002). Researching Aboriginal health: Experience from a study of urban young people's health and well-being. Social Science \& Medicine, 54, 1267-1279. doi:10.1016/S0277-9536(01)00095-8

Jamieson, L. M., Paradies, Y. C., Eades, S., Chong, A., Maple-Brown, L., Morris, P., Brown, A., et al. (2012). Ten principles relevant to health research among Indigenous Australian populations. The Medical Journal of Australia, 197, 16-18. doi:10.5694/mja11.11642

Johnson, C. A. ( 1986). Prevention and control of drug abuse. In J. M. Last (Ed.), Maxcy-Rosenau public health and preventive medicine (pp. 1075-1087). Norwalk, CT: Appleton-Century-Crofts.

Kirmayer, L. J., \& Valaskakis, G. G. (2009). Healing traditions: The mental health of Aboriginal peoples in Canada. Vancouver: UBC Press.

Limb, G. E., \& Hodge, D. R. (2011). Utilizing spiritual ecograms with Native American families and children to promote cultural competence in family therapy. Journal of Marital and Family Therapy, 37, 81-94. doi:10.1111/j.1752-0606.2009.00163.x

O’Neil, M., Pederson, A., Dupéré, S., \& Rootman, I. (2007). Health promotion in Canada: Critical perspectives (2nd ed.). Toronto: Canadian Scholar's Press.

Rice, W. (2011). Health promotion through an equity lens: Approaches, problems and solutions. Wellesley Institute. URL. http://site.ebrary.com/lib/unbc/Doc?id=10478581\&ppg=2

Selltiz, C., Jahoda, M. L., Deutsch, M., \& Stuart, S. W. (1967). Research methods in social relations. Toronto, ON: Holt, Rinehart and Winston.

Smith, L. T. (1999). Decolonizing methodologies: Research and indigenous peoples. London: Zed Books.

Turnbull, A. P., Friesen, B. J., \& Ramirez, C. (1998). Participatory action research as a model for conducting family research. Research and Practice for Persons with Disabilities, 23, 178-188. doi:10.2511/rpsd.23.3.178

Walker, R., Cromarty, H., Kelly, L., \& St Pierre-Hansen, N. (2009). Achieving cultural safety in Aboriginal health services: Implementation of a cross-cultural safety model in a hospital setting. Diversity in Health and Care, 6, 11-22. 